\title{
Article \\ Carbapenem-Resistant Klebsiella pneumoniae Clinical Isolates: In Vivo Virulence Assessment in Galleria mellonella and Potential Therapeutics by Polycationic Oligoethyleneimine
}

\author{
Dalila Mil-Homens ${ }^{1} \mathbb{1}$, Maria Martins ${ }^{1}$, José Barbosa ${ }^{1}$, Gabriel Serafim ${ }^{1}$, Maria J. Sarmento ${ }^{2} \mathbb{D}$, Rita F. Pires ${ }^{1}(0$, \\ Vitória Rodrigues ${ }^{3}$, Vasco D.B. Bonifácio ${ }^{1, *(D)}$ and Sandra N. Pinto ${ }^{1, *(D)}$ \\ 1 iBB-Institute for Bioengineering and Biosciences, Department of Bioengineering, Instituto Superior Técnico, \\ Universidade de Lisboa, Av. Rovisco Pais, 1049-001 Lisboa, Portugal; \\ dalilamil-homens@tecnico.ulisboa.pt (D.M.-H.); mald.martins@campus.fct.unl.pt (M.M.); \\ josembarbosa@tecnico.ulisboa.pt (J.B.); gabriel.serafim@tecnico.ulisboa.pt (G.S.); \\ ritafpires@tecnico.ulisboa.pt (R.F.P.) \\ 2 J. Heyrovský Institute of Physical Chemistry of the Czech Academy of Sciences, Dolejskova 3, \\ 18223 Prague, Czech Republic; maria.sarmento@jh-inst.cas.cz \\ 3 Seção de Microbiologia, Laboratório SYNLAB-Lisboa, Grupo SYNLAB Portugal, \\ Av. Columbano Bordalo Pinheiro, 75 A, $2^{\circ}$ Andar, 1070-061 Lisboa, Portugal; vitoria.rodrigues@synlab.pt \\ * Correspondence: vasco.bonifacio@tecnico.ulisboa.pt (V.D.B.B.); sandrapinto@ist.utl.pt (S.N.P.)
}

Citation: Mil-Homens, D.; Martins, M.; Barbosa, J.; Serafim, G.; Sarmento, M.J.; Pires, R.F.; Rodrigues, V.; Bonifácio, V.D.; Pinto, S.N. Carbapenem-Resistant Klebsiella pneumoniae Clinical Isolates: In Vivo Virulence Assessment in Galleria mellonella and Potential Therapeutics by Polycationic Oligoethyleneimine. Antibiotics 2021, 10, 56. https:// doi.org/10.3390/antibiotics10010056

Received: 30 September 2020

Accepted: 6 January 2021

Published: 8 January 2021

Publisher's Note: MDPI stays neutral with regard to jurisdictional clai$\mathrm{ms}$ in published maps and institutional affiliations.

Copyright: $(\odot 2021$ by the authors. Licensee MDPI, Basel, Switzerland. This article is an open access article distributed under the terms and conditions of the Creative Commons Attribution (CC BY) license (https:// creativecommons.org/licenses/by/ $4.0 /)$.
Abstract: Klebsiella pneumoniae, one of the most common pathogens found in hospital-acquired infections, is often resistant to multiple antibiotics. In fact, multidrug-resistant (MDR) K. pneumoniae producing KPC or OXA-48-like carbapenemases are recognized as a serious global health threat. In this sense, we evaluated the virulence of K. pneumoniae $\mathrm{KPC}(+)$ or OXA-48(+) aiming at potential antimicrobial therapeutics. K. pneumoniae carbapenemase (KPC) and the expanded-spectrum oxacillinase OXA-48 isolates were obtained from patients treated in medical care units in Lisbon, Portugal. The virulence potential of the K. pneumonia clinical isolates was tested using the Galleria mellonella model. For that, G. mellonella larvae were inoculated using patients $\mathrm{KPC}(+)$ and OXA-48(+) isolates. Using this in vivo model, the $\mathrm{KPC}(+) \mathrm{K}$. pneumoniae isolates showed to be, on average, more virulent than OXA-48(+). Virulence was found attenuated when a low bacterial inoculum (one magnitude lower) was tested. In addition, we also report the use of a synthetic polycationic oligomer (L-OEI-h) as a potential antimicrobial agent to fight infectious diseases caused by MDR bacteria. L-OEI-h has a broad-spectrum antibacterial activity and exerts a significantly bactericidal activity within the first 5-30 min treatment, causing lysis of the cytoplasmic membrane. Importantly, the polycationic oligomer showed low toxicity against in vitro models and no visible cytotoxicity (measured by survival and health index) was noted on the in vivo model (G. mellonella), thus L-OEI-h is foreseen as a promising polymer therapeutic for the treatment of MDR K. pneumoniae infections.

Keywords: Klebsiella pneumoniae; KPC and OXA-48-like carbapenemases; Galleria mellonella infection model; linear oligoethyleneimine hydrochloride

\section{Introduction}

The widespread use of antibiotics in clinics caused an increased frequency of multidrugresistance bacteria mainly due to bacterial mutations [1]. In particular, the emergence of resistance to last resource antibiotic treatment options (including carbapenems) has contributed to the limitation of effective therapeutics. Recent reports revealed a weak pipeline for novel antibiotics. From all the compounds under development, very few target infections were caused by Gram-negative bacteria [2-4]. This is clinically relevant, as Gram-negative bacteria infections are significantly more lethal compared to those caused by the Gram-positive [3]. Several factors contribute to the scarcity of new antibiotics, market 
failure being the most relevant. As result of a low return investment, pharmaceutical companies lack incentives for novel antibiotics development. Antibiotics are fast-acting drugs (limiting patient requirements to a small-time window) and the use of novel antibiotics is often reserved since, ultimately, an unpredictable resistance may occur $[1,5]$. Therefore, the development of novel and efficient antimicrobial agents is of utmost priority.

Klebsiella pneumonia, a pathogen of the Enterobacteriaceae family, is resistant to last resource antibiotics and is the source of some of the most complicated hospital-acquired infections [6-8]. Resistance to carbapenem in K. pneumonia poses a significant threat to patients in hospitals as this organism can cause life-threatening infections such as pneumonia, bloodstream infections and sepsis [9]. Several factors are associated with the acquisition of K. pneumonia $\mathrm{KPC}(+)$ and OXA-48(+) bacteria, including prolonged hospitalization, infections caused by medical devices (including contamination of ventilators and catheters) and overuse of antibiotics (e.g., carbapenems). Carbapenems-resistant K. pneumonia bacteria are capable of inactivating carbapenems via the production of carbapenemase enzymes. Several carbapenemases have been identified and categorized into classes. The ambler classes A (KPC, plasmid-mediated clavulanic acid-inhibited $\beta$-lactamases) and D (OXA-48, expanded-spectrum oxacillinase) categories are considered relevant carbapenemases, being highly resistant to all $\beta$-lactam molecules, including carbapenems [10].

To investigate the in vivo relevance of MDR K. pneumonia infection, we obtained different $\mathrm{KPC}(+)$ and OXA-48(+) isolates and determined their virulence using Galleria mellonella, a caterpillar model of infection. The success of MDR K. pneumonia infections depends, "among other factors", on the ability of the pathogen to escape the host's defense mechanisms. The larvae of the greater wax moth G. mellonella have been successfully employed as a model host to study virulence of human pathogenic agents, including several human pathogens, and to investigate the efficacy of therapeutic drugs [11-14]. G. mellonella possess only an innate immune system (that includes melanization, hemolymph, and several antimicrobial peptides). However, this is enough to offer powerful resistance to microbial infections [15]. Additionally, their innate immune system shares a high degree of structural and functional homology with the innate immune systems of mammal's [16]. Thus, evaluation of G. mellonella responses to K. pneumonia isolates infection can provide indication of the mammalian response to these pathogens.

Very few treatment options are available for patients infected with K. pneumoniae producing KPC or OXA-48-like carbapenemases and are often limited to administration of multiple antibiotic therapies and to colistin $[17,18]$. In the light of this, in this study, we report the use of a polycationic synthetic oligomer, linear oligoethyleneimine hydrochloride (L-OEI-h), as an antimicrobial agent for the treatment of K. pneumonia KPC $(+)$ and OXA$48(+)$ bacterial infections. We have previously reported the synthesis and biocidal activity of L-OEI-h against Streptococcus aureus and Escherichia coli [19]. Herein, we evaluate L-OEI-h antibacterial activity against MDR bacteria clinical isolates, namely K. pneumoniae, and investigate the underlying mechanism of action, which, as found for other polycationic antimicrobial agents, might involve disruption of the cell wall and/or the disintegration of the cytoplasmic membrane [10].

\section{Results}

\subsection{Evaluation of K. pneumoniae Virulance in G. mellonella Infection Model}

Ten K. pneumoniae isolates (Table S1) with reduced sensitivity to carbapenems were obtained from different clinical specimens. To investigate the virulence of K. pneumoniae isolates in vivo, we used the G. mellonella infection model (Figure 1). In this case, larvae survival rates were measured by injecting inoculums, incubating at $37^{\circ} \mathrm{C}$ and recording the survival rate daily for up to three days. In all experiments, control groups with administration of phosphate-buffered saline (PBS) solution resulted in a $100 \%$ survival rate. 


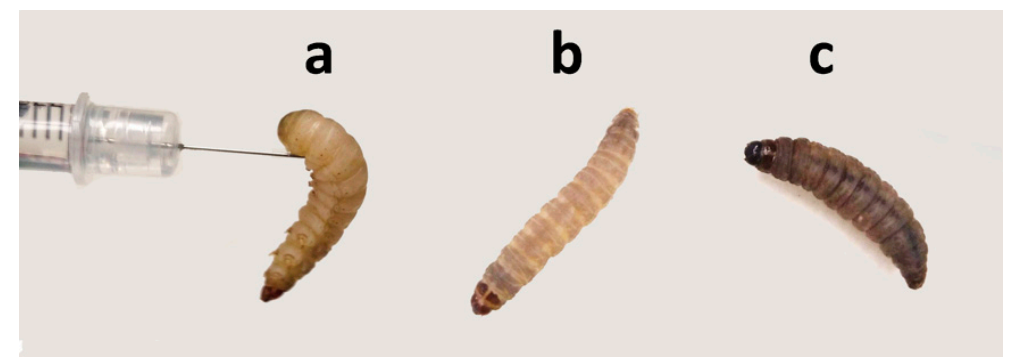

Figure 1. In vivo assays using the Galleria mellonella larva model. Inoculation by injection of different bacteria inoculum (a), healthy larva (b), and dead larva (c) as a result of Klebsiella pneumoniae infection.

Most larvae were found healthy following infection with $1 \times 10^{4} \mathrm{CFU}$ (colony-forming unit) of each isolate per larva (Figure S1), which suggests that at this bacterial density the humoral immunity of the insects is enough to produce an adequate response to the infection. However, the larvae survival was significantly altered upon increase of infection ratio $\left(1 \times 10^{5} \mathrm{CFU}\right.$ per larva). As shown in Figure 2, the virulence of isolates varied widely, with some $\mathrm{KPC}(+)$ isolates promoting total larvae mortality (e.g., SYN7 KPC clinical isolate). These differences in virulence are in accordance with data reported for patients suffering from K. pneumoniae $\mathrm{KPC}(+)$ infections [20], confirming the reliability of the G. mellonella infection model in reporting pathogenicity differences between all K. pneumoniae isolates. This infection model (G. mellonella infected with $1 \times 10^{5}$ CFU K. pneumoniae per larva) is now well established in our lab, and we believe that will be very helpful in future development of K. pneumoniae therapeutics.

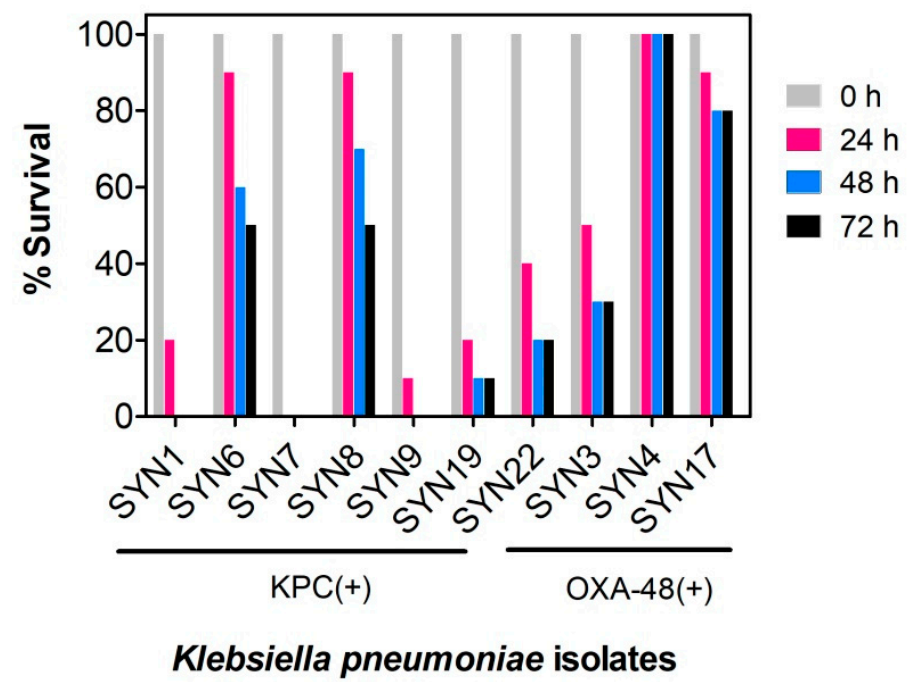

Figure 2. Evaluation of virulence of Klebsiella pneumoniae $\mathrm{KPC}(+)$ and $\mathrm{OXA}-48(+)$ isolates in Galleria mellonella. Survival of $G$. mellonella was followed for three days after infection with K. pneumoniae $\mathrm{KPC}(+)$ and OXA-48(+) with $1 \times 10^{5} \mathrm{CFU}$ per larva. Ten larvae were analyzed in each condition and larvae survival was monitored daily. In all cases, no larvae death was observed upon administration of PBS (control). KPC(+) isolates: SYN1, SYN6, SYN7, SYN8, SYN9 SYN19 and SYN22; OXA-48(+) isolates: SYN3, SYN4 and SYN17.

\subsection{Antimicrobial Activity of L-OEI-h}

The oligomer L-OEI-h was synthesized following our reported protocol [19]. We evaluated the antimicrobial activity of L-OEI-h against K. pneumoniae isolates by determination of the minimum inhibitory concentration (MIC) and the minimum bactericidal concentration (MBC). MIC is defined as the lowest drug concentration that prevents visible growth of bacteria. The $\mathrm{MBC}$ is the lowest concentration of an antimicrobial agent required to kill $\geq 99.9 \%$ bacteria over an extended period $(18-24 \mathrm{~h})$. The antimicrobial assays were 
conducted according with CLSI guidelines in Mueller Hinton broth (MHB), a nutrient rich bacterial growth medium. The obtained results for the different isolates are shown in Table 1. We included other Gram-negative bacteria (Pseudomonas aeruginosa PAO and E. coli AB1157) and a Gram-positive strain (S. aureus MRSA JE2) as control strains. Except for SYN7 KPC, which is also the most virulent isolate, L-OEI-h displayed good antibacterial activity against Gram-negative strains with particular relevance for P. aeruginosa PAO and E. coli AB1157. The MIC and MBC values were almost identical, which is indicative that the oligomer exerts a bactericidal activity.

Table 1. Antimicrobial activity of linear oligoethyleneimine hydrochloride (L-OEI-h) against Klebsiella pneumoniae isolates, control bacterial strains (P. aeruginosa PAO, E. coli AB1157) and the methicillinresistant Staphylococcus strain S. aureus JE2.

\begin{tabular}{ccc}
\hline Clinical Isolate & $\begin{array}{c}\text { MIC } \\
(\mu \mathbf{g} / \mathbf{m L})\end{array}$ & $\begin{array}{c}\text { MBC } \\
(\mu \mathbf{g} / \mathbf{m L})\end{array}$ \\
\hline SYN1 KPC & 458 & 458 \\
SYN3 OXA-48 & 915 & $>915$ \\
SYN4 OXA-48 & 458 & 915 \\
SYN6 KPC & 915 & 915 \\
SYN7 KPC & $>915$ & $>915$ \\
SYN8 KPC & 458 & 458 \\
SYN9 KPC & 229 & $229-458$ \\
SYN17 OXA-48 & 915 & 915 \\
SYN19 KPC & 915 & 915 \\
SYN22 KPC & 915 & $>915$ \\
P. aeruginosa PAO & 114 & 114 \\
E. coli AB1157 * & 90 & 90 \\
S. aureus JE2 & $>915$ & $>915$ \\
\hline
\end{tabular}

${ }^{*}$ Data acquired in a previous study [13].

We previously demonstrated that L-OEI-h is also able to target some Gram-positive bacteria with low efficiency [19], as demonstrated here for methicillin-resistant S. aureus JE2 (MIC $>915 \mu \mathrm{g} / \mathrm{mL})$.

\subsection{Biocompatibility Studies}

Some membrane-lytic agents are known to display selectivity towards bacterial membranes, allowing for elevated antibiotic activity and low toxicity to mammalian cells [21]. The differences in composition and lipid arrangement in bacterial and mammalian cell membranes can support the selectivity observed in these antimicrobial agents [21,22]. Here, we evaluated the cytotoxicity of L-OEI-h using in vitro (L929 mouse fibroblasts) and in vivo models (G. mellonella larvae) (Figure 3).

The polycationic oligomer compound has very little cytotoxicity against mammalian cell lines, even at the highest dose tested against clinical isolates $(915 \mu \mathrm{g} / \mathrm{mL}$, Figure 3a,b). The larvae were injected with $5 \mu \mathrm{L}$ of different concentrations of L-OEI-h, and then incubated in Petri dishes at $37^{\circ} \mathrm{C}$ and daily scored for survival. Up to a concentration of $915 \mu \mathrm{g} / \mathrm{mL}$, all larvae were found healthy for a three-day period (Figure 3c). To obtain more differences in larvae health, we also determined the health index scores (Figure 3d), which scores four main parameters: larvae activity, cocoon formation, melanization and survival. The injection of the larvae with L-OEI-h even after $72 \mathrm{~h}$ resulted in high health index scores. The higher activity and more cocoon formation are regularly associated to a healthier wax worm [23].

All experiments included a control group injected only with a PBS solution. Overall, our results corroborate the biocompatibility of L-OEI-h. 
a

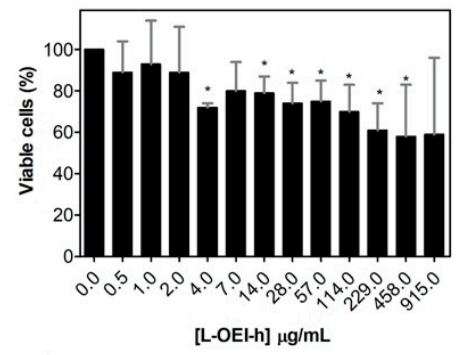

C

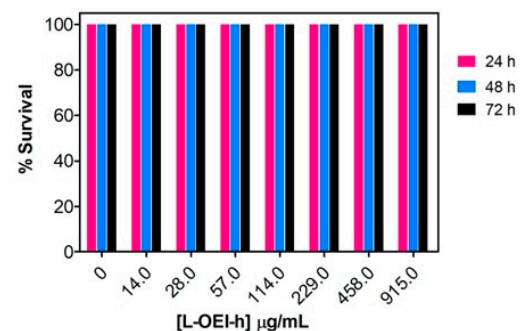

b

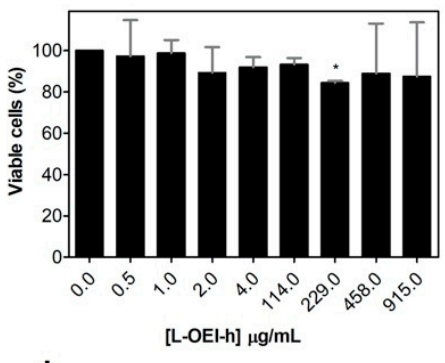

d

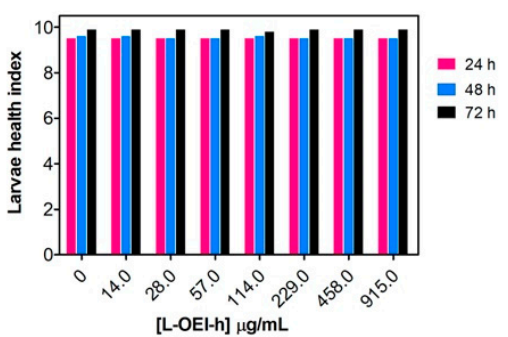

Figure 3. Evaluation of linear oligoethyleneimine hydrochloride (L-OEI-h) toxicity and biocompatibility using in vitro mammalian cells (L929 mouse and A549 human epithelial cells) (a,b) and in vivo assays (Galleria mellonella larvae) (c). The health index scores of wax worms injected with L-OEI-h was also evaluated $(\mathbf{d})$. Asterisks $\left({ }^{*}\right)$ represent statistical significance in $t$-student tests $(p<0.05)$ compared to the untreated samples.

\subsection{Exploring the L-OEI-h Mechanism of Action}

Cationic polymers are expected to target the microbial cell surface, via binding to negatively charged components, and to disrupt the cytoplasmic membrane [24]. A fastkilling kinetics is associated with surface membranolytic processes, while slow kinetics is usually associated with activation of intracellular processes [25-28]. In this sense a time-kill assay based on traditional colony count was carried out to discriminate between fast and slow kinetics of antibacterial activity.

Time-kill curves of a selected OXA-48(+) (SYN4 OXA-48) and KPC(+) isolates (SYN8 $\mathrm{KPC}$ ) were obtained in the presence of two different oligomer concentrations. As shown in Figure 4 and Figure S2, after the addition of L-OEI-h, the number of K. pneumoniae viable and culturable colonies decreases significantly in the first $30 \mathrm{~min}$ of treatment, with almost complete bacterial removal achieved at $2 \mathrm{~h}$ treatment. This observation illustrates a possible fast L-OEI-h bactericidal activity. For other Gram-negative bacteria (e.g., E. coli AB1157 [19]), L-OEI-h had a very fast killing effects (within $5 \mathrm{~min}$ ). In both cases, the verified fast activity is supportive of a surface membranolytic mechanism of action for L-OEI-h.

a

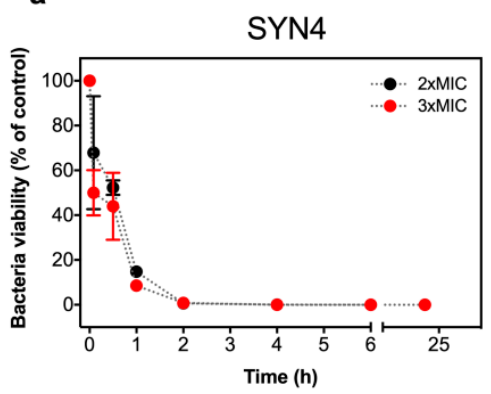

b

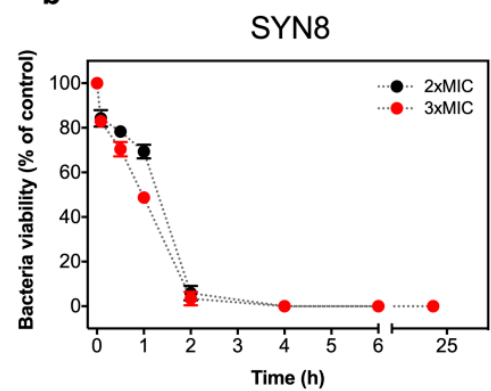

Figure 4. Killing kinetics of Klebsiella pneumonia SYN4 OXA-48 (a) and SYN8 KPC (b) clinical isolates induced by L-OEI-h. The killing kinetics was evaluated with a colony count assay using two different oligomer concentrations, $2 \times$ MIC and $3 \times$ MIC. 
The higher antimicrobial activity of L-OEI-h against Gram-negative bacteria (e.g., E. coli AB1157 vs. S. aureus NCTC8325-4 [19]) may indicate that the presence of lipopolysaccharides (LPS) in the outer leaflet of the outer membrane of Gram-negative may facilitate the initial binding of the compound. Antibacterial cationic polymers are expected to permeabilize bacterial membrane by one of two possible mechanisms: (i) Perpendicular insertion in the membrane followed by pore formation and membrane permeabilization/depolarization, or (ii) accumulation at the membrane surface until a certain threshold concentration is achieved, which leads to membrane disruption and cell lysis.

To further characterize the mechanism of action of L-OEI-h, studies with membrane mimetics were carried out. In these simplified membrane models, size, geometry, and composition can be tailored with great precision.

The effect of L-OEI-h on the membrane was thus, studied using large unilamellar vesicles (LUVs) of controlled lipid composition, serving as mimetics of bacterial and mammalian cell membranes. The bacterial membrane model, with an overall negative charge, was composed of different ratios of phosphatidylcholine (POPC) and phosphatidylglycerol (POPG), while the healthy mammalian plasma membrane model contained only POPC [20]. POPC zwitterionic liposomes are not affected by the addition of L-OEI-h, but higher amount of negatively charged lipids (>20\%) led to the formation of large clusters within a few seconds (Figure 5). We hypothesize that the formation of large clusters $(>100 \mathrm{~nm})$ is consistent with the binding/accumulation of the polymer in membranes with high negatively charged lipids.

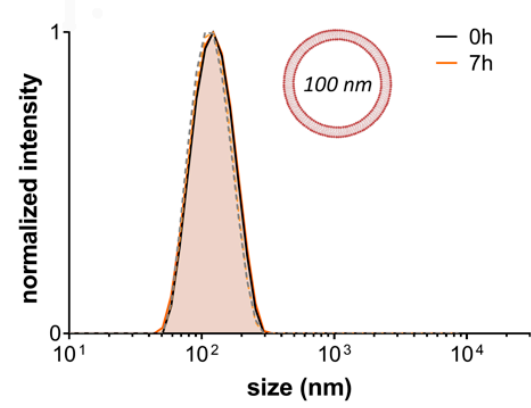

b

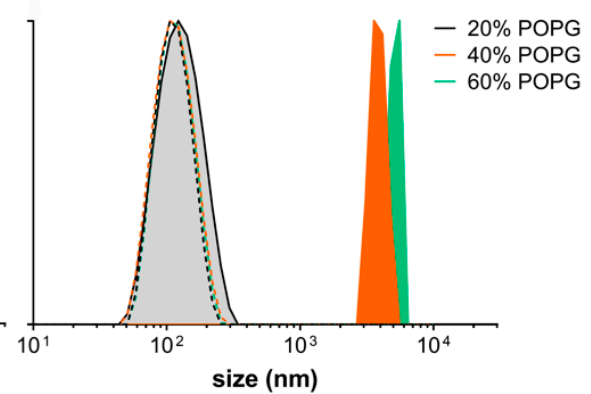

Figure 5. Effect of L-OE-h on model liposomes composed of phosphatidylcholine (POPC) (a) and POPC with varying phosphatidylglycerol (POPG) content (b). Vesicle sizes were measured by DLS at $25^{\circ} \mathrm{C}$. Dashed lines are the respective controls without polymer.

Upon electrostatic binding of L-OEI-h to anionic lipid vesicles, and after a certain threshold concentration, the oligomer induces vesicle aggregation and/or vesicle fusion, which explains the appearance of a LUV population larger in size (Figure 5b). Vesicle aggregation and fusion are not unusual occurrences, being also observed in the case of cationic peptides addition to anionic vesicles [29]. Ultimately, accumulation of L-OEI-h in the membrane may induce micelle formation or the formation of transient pores, as illustrated for the mechanism of action of some antimicrobial peptides (e.g., [29]). In both cases membrane disintegration is a consequence of these events (Figure 6). 


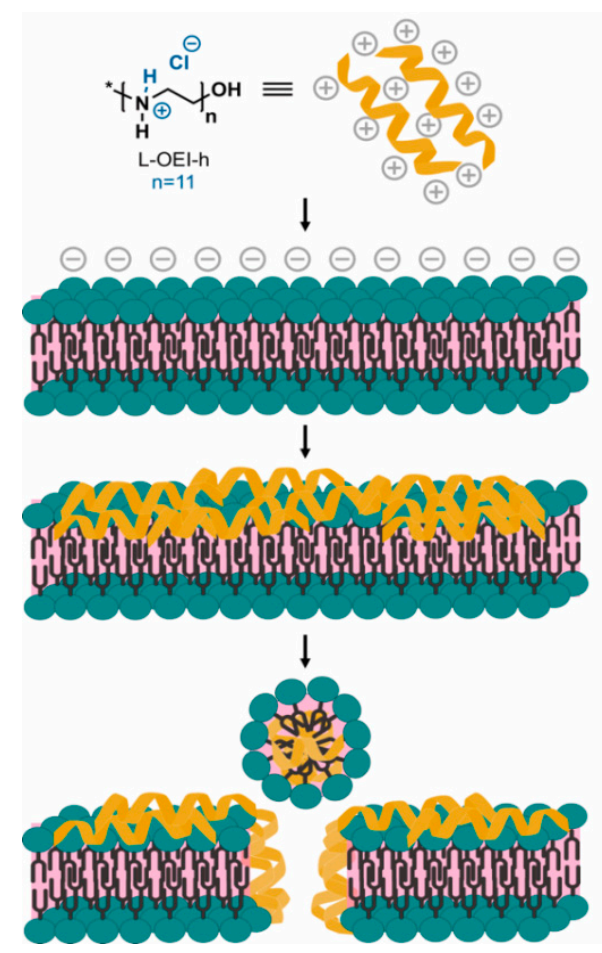

Figure 6. Hypothetical mechanism of action of L-OEI-h (model II) towards bacterial cell membranes. The accumulation of the polycationic oligomer at the membrane surface, due to electrostatic interactions, results in an oligomer threshold concentration capable of cell disruption and lysis.

\section{Discussion}

Bacterial infections are becoming a major human health problem. Resistance towards antibiotics is becoming increasingly common, including to the ones only reserved for the treatment of severe infections. The World Health Organization has recently included Klebsiella in the critical list of microorganisms for which new therapeutics are urgently needed [30]. In the light of this demand, in this study we examine the sensitivity of G. mellonella to K. pneumoniae isolates that are resistant to last resources antibiotics and focused our attention towards a polycationic oligomer [19], a synthetic mimic of host defense peptides (HDPs), as a novel treatment for MDR K. pneumonia infections.

HDPs are a class of innate immunity components expressed by all multicellular organisms [31]. It is believed that their function is, in part, to kill invasive cells without prejudice to the host and without presenting itself as a stress agent for the development of resistance traits [31]. The discovery of HDPs was accompanied by the development of disinfectant polymers, which in the late 1990s led to HDP-mimicking polymers [32]. Although antimicrobial peptides (AMPs)/HDPs are an excellent alternative to conventional antibiotics, cheap and scalable bioprocessing is not yet available [33]. Additionally, AMPs are poorly stable in vivo due to protease liability.

In this way, HDP-mimicking polymers are considered a more cost-effective and stable alternative to HDP / AMP [31]. The relationship between structure and activity of HDPmimetic polymers relies on two main design principles: (i) Hydrophobic/hydrophilic component ratio and (ii) presence of a structured cationic group. It was demonstrated that a poly(methacrylate) random copolymer with $40 \%$ methyl side chains (hydrophobic segment) and $60 \%$ aminoethyl side chains (hydrophilic segment) show potent antibacterial activity and low hemolytic activity [34].

The effect of primary amine groups, instead of traditional quaternary ammonium salt (QAS), was already investigated [35]. For the same type of polymer, primary amines outperformed in comparison with tertiary or quaternary amines in terms of antimicrobial activity and toxicity. Following this study, it was found that primary ammonium groups can form a stronger complex with phospholipid headgroups when compared to QAS analogues [36]. 
Additionally, the effect of amine groups density is quite relevant since increasing the amine density by monomer unit enhances the polymers efficacy and decreases hemolysis in great extent [37].

The synthetic polymer polyethyleneimine (PEI), due to its intrinsic features, is regarded as a good alternative to fight antibiotic resistant organisms. The abundance of reactive amine groups in the backbone allows post-modifications to display both hydrophobicity and a positive charge density, primary requirements for a good antimicrobial activity [38]. In previous studies, we found that L-OEI-h, a PEI analogue, is a very effective biocidal oligomer [19], whose activity may be attributed to high positive charge density (hydrochloride salt, quaternary nitrogen atoms), if compared with commercial L-PEI (nonquaternary nitrogen atoms) [39] (see Figure 7). As we and others demonstrated, a higher positive charge density leads to higher antimicrobial efficacy [40].

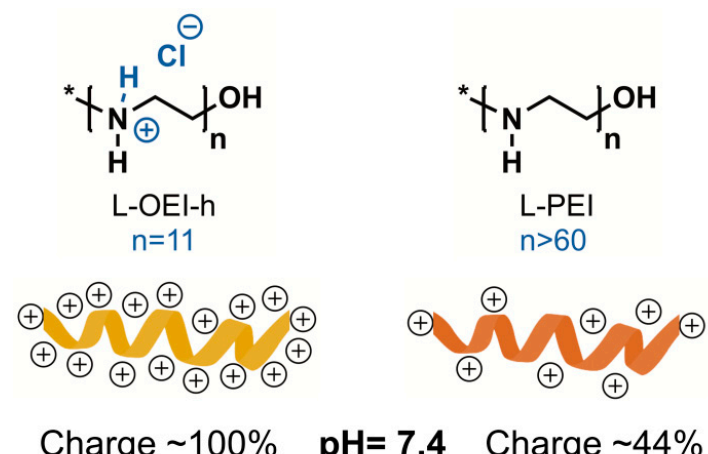

Figure 7. Comparison of positive charge density between linear oligoethyleneimine hydrochloride (L-OEI-h) and linear polyethyleneimine (L-PEI) at physiological $\mathrm{pH}$.

In this work, we evaluated the antimicrobial activity of L-OEI-h against a variety of K. pneumonia strains. The antimicrobial efficiency is attributed to favorable electrostatic interactions between the polycationic oligomer (having $c a .+11$ formal net charge, one positive charge per monomer unit) and the anionic bacterial membrane. This interaction can induce a very fast-bactericidal activity, as demonstrated by us [19]. Such a fast mode of action strongly suggests that membranolytic processes are responsible for antimicrobial activity. Through direct permeabilization of the lipidic membrane, instead of action on a specific cellular target, the probability of bacteria to develop resistance to this treatment is extremely low.

For K. pneumoniae clinical isolates, the bactericidal activity of the oligomer does not occur earlier than $30 \mathrm{~min}$, in contrast with what we and others verified for other membrane disruption agents, whereby membrane permeabilization and/or lysis happened within 5 min $[19,41,42]$. It is likely that, for K. pneumoniae clinical isolates, L-OEI-h cannot diffuse so efficiently (or diffuses slowly) through the bacterial cell wall to reach the plasma membrane. This could be associated with the fact that the capsule (composed of extracellular polysaccharides) of K. pneumoniae is more "robust" than what was verified for other gram-negative bacteria, and, in this sense, constitutes an efficient barrier against several antimicrobial agents $[43,44]$ including HDPs. Despite this, L-OEI-h was able to efficiently kill several $K$. pneumoniae clinical isolates (Table 1, Figure 4 and Figure S2).

Importantly, the L-OEI-h antimicrobial action against some K. pneumoniae clinical isolates such as SYN4 OXA-48 and SYN9 KPC (a highly virulent clinical isolate) is verified under a possible therapeutic window, since L-OEI-h induces low cytotoxicity against in vitro mammalian cell lines $(<80 \%$ cell viability [45] within statistical error) and, more importantly, no visible cytotoxicity was detected against the in vivo model (G. mellonella). As shown here, low toxicity in these models is likely associated with reduced interaction with the plasma membrane of eukaryotic cells. Liposomes with a lipid composition mimicking the outer leaflet of eukaryotic plasma membranes were not affected by the presence of L-OEI-h (Figure 5a). On the other hand, liposomes rich in phosphatidylglycerol, 
mimicking bacterial membrane lipid composition, showed dramatic aggregation upon interaction with this compound. Large unilamellar vesicles (LUVs) were used in these studies as their size is more amenable to DLS resolution. Other membrane models, such as multilamellar vesicles (MLVs), due to their multi-layered character, prevent polymer interactions with the internal bilayers (that could mask possible effects occurring only in the outer membrane), while small unilamellar vesicles (SUV)'s small size results in membranes with excessive curvature that do not mimic bacterial membranes. On the other hand, very large vesicles such as giant unilamellar vesicles (GUVs) have a less controlled lipid concentration/size and thus frequently show significant vesicle heterogeneity within the same sample.

Although we obtained promising data for some K. pneumoniae isolates, we verified that in some cases MIC and MBC values were still high (if compared with the results obtained for the Gram-negative control strains). The polymer design and the polymermembrane interactions are crucial issues for bacterial infection eradication. In a recent study, linear and branched PEI polymers were found to have very similar MIC values, while $\varepsilon$ polycaprolactone showed superior broad-spectrum antimicrobial properties over L-PEI [46]. Hence, in future work we will consider the use of a coarse-grain (CG) molecular dynamics model of L-OEI-h to better understand its interactions with bacterial and mammalian membranes. This study will allow the identification of key oligomer-membrane interactions which could lead to enhanced polymer antimicrobials.

\section{Materials and Methods}

\subsection{Synthesis of Linear Oligoethyleneimine Hydrochloride (L-OEI- $h$ )}

The preparation of L-OEI-h was made in two steps following our previous protocol. First, linear 2-ethyl(2-oligooxazoline) (OEtOx) was synthesized by cationic ring-opening polymerization (CROP) in supercritical carbon dioxide, a green polymerization methodology [47]. The living OEtOx polymer was terminated with water and isolated as a brownish sticky oil. Next, OEtOx was hydrolyzed overnight using a $\mathrm{HCl} 5 \mathrm{M}$ solution. After this period, the precipitated solid was filtered and washed with acetone to obtain L-OEI-h as an off-white solid in quantitative yield. After vacuum drying, L-OEI-h is ready to use [19].

\subsection{Clinical Isolates Collection and Identification}

The KPC and OXA-48-positive carbapenems-resistant K. pneumoniae clinical isolates were collected from patients treated in medical care units (Lisbon, Portugal). All strains were identified with matrix-assisted laser desorption/ionization time-of-flight (MALDITOF) spectrometry [48,49] using the VITEK MS system (bioMérieux). Briefly, inoculation loops were used to select and smear the isolates onto the sample spots/target slide. Then $1 \mu \mathrm{L}$ VITEK mass spectrometry $\alpha$-cyano-4-hydroxycinnamic acid (MS-CHCA) matrix was applied over the sample and air dried (1-2 min). The target slide was loaded into the VITEK MS system to acquire the mass spectra of whole bacterial cell protein (which is mainly composed of ribosomal proteins). Then, the mass spectra acquired for each sample were compared to the mass spectra contained in the database.

In addition, the following antimicrobials were included in the microorganism's characterization: $\beta$-lactams (ceftazidime, cefepime, cefuroxime/axetil, amoxicillin/clavulanate, ticarcillin/clavulanate and piperacillin/tazobactam), carbapenems (meropenem, ertapenem), aminoglycosides (gentamicin, amikacin), nitrofurantoin fluoroquinolones (ciprofloxacin), polymyxin (colistin), fosfomycin, trimethoprim/sulfamethoxazole. The production of carbapenemases in these strains is evidenced through the antibiotic resistance profile and the type of carbapenemase (OXA-48-like, KPC, NDM or VIM) was identified through an immunochromatographic method (RESIST-4 O.K.N.V., Coris). CASFM-EUCAST 2016defined breakpoints for Enterobacteriaceae were used to interpret susceptibility data for $K$. pneumoniae (http:/ / www.sfm-microbiologie.org).

From pure culture on MacConkey agar plates, all identified K. pneumoniae isolates were transferred to $1.5 \mathrm{~mL}$ Eppendorf tubes contain Luria-Bertani (LB) broth with 20\% 
$(v / v)$ glycerol and were maintained at $-80^{\circ} \mathrm{C}$ for long-term storage. For the antimicrobial activity studies, bacterial cultures were inoculated in Mueller-Hinton broth (MHB) (Difco), at $37^{\circ} \mathrm{C}$. Tryptic soy agar (TSA) agar plates (bioMérieux, Marcy l'Etoile, France) were used to subculture K. pneumoniae isolates, P. aeruginosa PAO and E. coli AB1157. S. aureus MRSA JE2 was streaked on Columbia agar $+5 \%$ sheep blood plates (COS, bioMérieux, Marcy $1^{\prime}$ Etoile, Auvergne-Rhône-Alpes, France) and grown overnight at $37^{\circ} \mathrm{C}$. P. aeruginosa PAO, E. coli AB1157 and S. aureus JE2 were used as reference strains. The antimicrobial activity of L-OEI-h against $E$. coli AB1157 was previously investigated by us [19].

\subsection{Galleria mellonella Infection Model}

G. mellonella wax moth larvae were reared in our lab at $25^{\circ} \mathrm{C}$ in the dark, from egg to last-instar larvae, on a natural diet (beeswax and pollen grains). Worms of the final-instar larval stage, weighing $250 \pm 25 \mathrm{mg}$, were selected for the experiments. The G. mellonella survival experiment was adapted from previous studies with small changes [11,14]. Briefly, all K. pneumoniae isolates were grown overnight in TSA plates. Then, the assay was carried out by preparing two distinct inoculums of $2 \times 10^{6}$ and $2 \times 10^{7} \mathrm{CFU} / \mathrm{mL}$ in PBS. Using a hypodermic microsyringe, the larvae were injected with $5 \mu \mathrm{L}$ of each bacterial suspension via the hindmost left proleg, previously surface sanitized with $70 \%(v / v)$ alcohol. Different groups were used ( $n=10$ each) -larvae injected with PBS to monitor the killing due to injection trauma (control) and larvae injected with K. pneumoniae isolates. After inoculation, larvae were kept in Petri dishes and maintained in the dark at $37^{\circ} \mathrm{C}$ for $72 \mathrm{~h}$. The larval survival was assessed daily during that period, and caterpillars were considered dead based on the lack of mobility in response to touch. Each larva was also scored daily to the G. mellonella health index, which scores four main parameters: Larvae activity, cocoon formation, melanization and survival, as described in [23].

All experiments were performed using a minimum of two independent experiments.

\subsection{Antimicrobial Activity}

\subsubsection{Minimum Inhibitory Concentration (MIC) Determination}

To determine the MIC values, the bacterial suspension was initially adjusted to a concentration of $1 \times 10^{6} \mathrm{CFU} / \mathrm{mL}$ in MHB (according to CLSI guidelines) [50]. On a 96-well plate (Orange Scientific, Braine-l'Alleud, Belgium), two-fold serial dilution (in MHB) of L-OEI-h was then added to each well that contained the bacterial inoculums (dilution 1:1). Final bacteria inoculum in each well were diluted to $5 \times 10^{5} \mathrm{CFU} / \mathrm{mL}$. The 96-well tissue culture plates were incubated for $18-20 \mathrm{~h}$ at $37^{\circ} \mathrm{C}$. All experiments were performed using a minimum of three independent experiments performed with three technical replicates each.

\subsubsection{Minimum Bactericidal Concentration (MBC) Determination}

The MBC values were determined by the traditional colony count assay [51]. At the end of the MIC assay, $20 \mu \mathrm{L}$ samples from each well (corresponding to $\frac{1}{2} \mathrm{MIC}$, MIC, $2 \times$ MIC, $3 \times$ MIC of L-OEI-h) were transferred to a new 96-well plate and successively diluted (10-fold) in MHB. Then, each dilution was sub-cultured in TSA plates. After incubation at $37^{\circ} \mathrm{C}$ for $24 \mathrm{~h}$, the resultant viable colonies were counted. All experiments were performed using a minimum of three independent experiments performed with three technical replicates each.

\subsubsection{In Vitro Time-Kill Curves}

Time-kill curves of L-OEI-h were determined according to literature [52,53]. Briefly, a final inoculum of $1 \times 10^{6}$ to $1 \times 10^{7} \mathrm{CFU} / \mathrm{mL}$ was exposed to distinct doses of L-OEI-h and incubated at $37^{\circ} \mathrm{C}$. Aliquots at specified time points were taken; 10-fold dilutions of each well were prepared and plated onto a TSA plate for CFU enumeration. TSA plates were incubated for $24 \mathrm{~h}$ at $37^{\circ} \mathrm{C}$ and bacterial colonies were counted. Viable cells $(\mathrm{CFU} / \mathrm{mL})$ are reported here as percentage of the control (bacterial suspension without 
L-OEI-h exposition). From the number of bacterial colonies obtained, viable bacteria (in $\mathrm{CFU} / \mathrm{mL}$ ) are reported as percentage of the control. All experiments were performed using two independent experiments with three technical replicates each.

\subsection{Biocompatibility Assays}

\subsubsection{MTT Viability Assay}

L-929 and A549 cell lines were cultured in T-75 cell culture flasks (Filter caps) using Dulbecco's modified Eagle's medium (DMEM, Catalog number 41966-029) supplemented with 10\% fetal bovine serum (Thermo Fisher Scientific, Catalog number 10500-064, heat inactivated) and $1 \%$ penicillin-streptomycin (Thermo Fisher Scientific, catalogue number 15140-122) and maintained in a humidified atmosphere with $5 \% \mathrm{CO}_{2}$ at $37{ }^{\circ} \mathrm{C}$. All cell culture lines were maintained with routinely subcultures (using TrypLE Express without phenol red, GIBCO ${ }^{\mathrm{TM}}$, for chemical detaching). Mammalian cell lines were counted with a hemocytometer.

The MTT assay was used to detect changes on metabolic activity of mammalian cells [54]. Briefly, the L-929 and A549 cell lines were seeded in 96-well flat-bottomed polystyrene plates with a density of $1 \times 10^{4}$ cells/well and left to adhere overnight in a $\mathrm{CO}_{2}$ incubator $(5 \%)$ at $37^{\circ} \mathrm{C}$. After $24 \mathrm{~h}$, the cell medium was discarded and replaced with fresh medium containing different concentrations of L-OEI-h. Cells were then incubated for a period of $24 \mathrm{~h}$ at $37^{\circ} \mathrm{C}$ in a humidified $5 \% \mathrm{CO}_{2}$ incubator. After this incubation period, the medium was discarded and $20 \mu \mathrm{L}$ of MTT $(5 \mathrm{mg} / \mathrm{mL})$ were added to each well together with $100 \mu \mathrm{L}$ of fresh DMEM and incubated at $37^{\circ} \mathrm{C}$ for $3.5 \mathrm{~h}$. The formazan crystals formed in the wells were dissolved using $150 \mu \mathrm{L}$ of MTT solvent $(4 \mathrm{mM}$ of $\mathrm{HCl}, 0.1 \%$ of Nondet P-40 in isopropanol). The formation of formazan was monitored by measuring the absorbance at $590 \mathrm{~nm}$ in a microplate reader (BMG Labtech, Polar Star Optima). Cell viability was determined relatively to the untreated sample after correcting the data with the negative control.

All experiments were performed using a minimum of two independent experiments with three technical replicates each.

\subsubsection{Galleria mellonella Toxicity Assay}

The L-OEI-h toxicity was also evaluated in the larvae infection in vivo model. The $G$. mellonella killing assays were based on the above descriptions with small modifications. L-OEI-h doses were prepared and injected into the larvae hindmost left proleg. The larvae survival was assessed daily during a period of $72 \mathrm{~h}$. A control group was also included in the assay. Two independent experiments were performed.

\subsection{Exploring the L-OEI-h Mechanism of Action}

Liposome Preparation

Large unilamellar vesicles (LUVs), with $100 \mathrm{~nm}$ of diameter, were prepared by extrusion of multilamellar vesicles [54]. The liposomes were prepared according to methods previously described [55]. Briefly, lipid mixtures composed of adequate amounts of lipids (POPC and POPG) were prepared in chloroform to a final lipid concentration of $2 \mathrm{mM}$. The solvent was slowly vaporized under a nitrogen flux and the resulting lipid film was left in vacuum for $3 \mathrm{~h}$ to ensure the complete removal of chloroform. Afterwards, the lipid was resuspended in $2 \mathrm{~mL}$ of DPBS (Thermo Fisher Scientific) and freeze-thaw cycles (liquid nitrogen/water bath at $60^{\circ} \mathrm{C}$ ) were performed to re-equilibrate and homogenize the samples. LUVs were finally obtained by extrusion of the solutions at $50{ }^{\circ} \mathrm{C}$ with an Avanti Mini-Extruder (Merck, Darmstadt, Germany) using $100 \mathrm{~nm}$ pore size polycarbonate membranes. All lipid stock solutions were prepared in chloroform and the respective concentrations were determined by the colorimetric quantification of inorganic phosphate.

Liposome size was determined by dynamic light scattering (DLS) using a Nanosizer ZS (Malvern Instruments). The POPC/POPG vesicles were incubated with L-OEI-h for 
5 min. Data was collected at $25^{\circ} \mathrm{C}$ and a backscattering angle of $173^{\circ}$. Two independent experiments were performed.

Supplementary Materials: The following are available online at https:/ /www.mdpi.com/2079-6 382/10/1/56/s1, Figure S1: Evaluation of distinct virulence response of K. pneumoniae KPC $(+)$ and OXA-48 (+) isolates in G. mellonella. Figure S2: Killing kinetics of K. pneumonia SYN4 OXA-48 (a) and SYN8 KPC (b) clinical isolates induced by L-OEI-h. Table S1: K. pneumonia clinical isolates origin and the minimum inhibitory concentration (MIC) values of trimethoprim (TM)/sulfamethoxazole (SM) antibiotics.

Author Contributions: Conceptualization, S.N.P., D.M.-H., V.D.B.B.; methodology, S.N.P., D.M.-H., V.D.B.B, R.F.P., M.M., J.B., M.J.S.; formal analysis, S.N.P., D.M.-H., V.D.B.B, M.J.S.; investigation, S.N.P., D.M.-H., R.F.P., M.M., J.B., M.J.S.; V.R.; writing—original draft preparation, S.N.P.; writing—review and editing, S.N.P., V.D.B.B, D.M.-H., M.J.S., G.S.; supervision, S.N.P., V.D.B.B. All authors have read and agreed to the published version of the manuscript.

Funding: This research was funded by Fundação para a Ciência e a Tecnologia (FCT, Portugal), Ministério da Ciência, Tecnologia e Ensino Superior (MCTES) through projects PTDC/MECONC/29327/2017, UIDB/04565/2020 and SAICTPAC/0019/2015.

Data Availability Statement: The data presented in this study are available in the article and in the supplementary material.

Conflicts of Interest: The authors declare no conflict of interest.

\section{References}

1. Aslam, B.; Wang, W.; Arshad, M.I.; Khurshid, M.; Muzammil, S.; Rasool, M.H.; Nisar, M.A.; Alvi, R.F.; Aslam, M.A.; Qamar, M.U.; et al. Antibiotic resistance: A rundown of a global crisis. Infect. Drug Resist. 2018, 11, 1645-1658. [CrossRef]

2. Coates, A.R.; Halls, G.; Hu, Y. Novel classes of antibiotics or more of the same? Br. J. Pharmacol. 2011, 163, 184-194. [CrossRef]

3. Breijyeh, Z.; Jubeh, B.; Karaman, R. Resistance of Gram-Negative bacteria to current antibacterial agents and approaches to resolve it. Molecules 2020, 25, 1340. [CrossRef]

4. Theuretzbacher, U.; Outterson, K.; Engel, A.; Karlén, A. The global preclinical antibacterial pipeline. Nat. Rev. Microbiol. 2020, 18, 275-285. [CrossRef]

5. Ventola, C.L. The antibiotic resistance crisis: Part 1: Causes and threats. PT 2015, 40, 277-283.

6. Nordmann, P.; Cuzon, G.; Naas, T. The real threat of Klebsiella pneumoniae carbapenemase-producing bacteria. Lancet Infect. Dis. 2009, 9, 228-236. [CrossRef]

7. Molton, J.S.; Tambyah, P.A.; Ang, B.S.; Ling, M.L.; Fisher, D.A. The global spread of healthcare-associated multidrug-resistant bacteria: A perspective from Asia. Clin. Infect. Dis. 2013, 56, 1310-1318. [CrossRef]

8. Tzouvelekis, L.S.; Markogiannakis, A.; Psichogiou, M.; Tassios, P.T.; Daikos, G.L. Carbapenemases in Klebsiella pneumoniae and other Enterobacteriaceae: An evolving crisis of global dimensions. Clin. Microbiol. Rev. 2012, 25, 682-707. [CrossRef] [PubMed]

9. Peleg, A.Y.; Hooper, D.C. Hospital-acquired infections due to Gram-negative bacteria. N. Engl. J. Med. 2010, 362, 1804-1813. [CrossRef]

10. Halat, D.H.; Moubareck, C.A. The current burden of carbapenemases: Review of significant properties and dissemination among Gram-negative bacteria. Antibiotics (Basel) 2020, 9, 186. [CrossRef]

11. Mil-Homens, D.; Bernardes, N.; Fialho, A.M. The antibacterial properties of docosahexaenoic omega-3 fatty acid against the cystic fibrosis multiresistant pathogen Burkholderia cenocepacia. FEMS Microbiol. Lett. 2012, 328, 61-69. [CrossRef] [PubMed]

12. Cutuli, M.A.; Petronio, G.P.; Vergalito, F.; Magnifico, I.; Pietrangelo, L.; Venditti, N.; Di Marco, R. Galleria mellonella as a consolidated in vivo model hosts: New developments in antibacterial strategies and novel drug testing. Virulence 2019, 10, 527-541. [CrossRef] [PubMed]

13. Jander, G.; Rahme, L.G.; Ausubel, F.M. Positive correlation between virulence of Pseudomonas aeruginosa mutants in mice and insects. J. Bacteriol. 2000, 182, 3843-3845. [CrossRef] [PubMed]

14. Mil-Homens, D.; Barahona, S.; Moreira, R.N.; Silva, I.J.; Pinto, S.N.; Fialho, A.M.; Arraiano, C.M. Stress response protein BolA influences fitness and promotes Salmonella enterica serovar Typhimurium virulence. Appl. Environ. Microbiol. 2018, 84, e02850-17. [CrossRef]

15. Vilmos, P.; Kurucz, E. Insect immunity: Evolutionary roots of the mammalian innate immune system. Immunol. Lett. 1998, 62, 59-66. [CrossRef]

16. Hoffmann, J.A. Innate immunity of insects. Curr. Opin. Immunol. 1995, 7, 4-10. [CrossRef]

17. Papst, L.; Beović, B.; Pulcini, C.; Durante-Mangoni, E.; Rodríguez-Baño, J.; Kaye, K.S.; Daikos, G.L.; Raka, L.; Paul, M. Antibiotic treatment of infections caused by carbapenem-resistant Gram-negative bacilli: An international ESCMID cross-sectional survey among infectious diseases specialists practicing in large hospitals. Clin. Microbiol. Infect. 2018, 24, 1070-1076. [CrossRef] 
18. Karakonstantis, S.; Kritsotakis, E.I.; Gikas, A. Treatment options for K. pneumoniae, P. aeruginosa and A. baumannii co-resistant to carbapenems, aminoglycosides, polymyxins and tigecycline: An approach based on the mechanisms of resistance to carbapenems. Infection 2020, 48, 835-851. [CrossRef]

19. Correia, V.G.; Bonifácio, V.D.B.; Raje, V.P.; Casimiro, T.; Moutinho, G.; da Silva, C.L.; Pinho, M.G.; Aguiar-Ricardo, A. Oxazolinebased antimicrobial oligomers: Synthesis by CROP using supercritical $\mathrm{CO}_{2}$. Macromol. Biosci. 2011, 11, 1128-1137. [CrossRef]

20. McLaughlin, M.M.; Advincula, M.R.; Malczynski, M.; Barajas, G.; Qi, C.; Scheetz, M.H. Quantifying the clinical virulence of Klebsiella pneumoniae producing carbapenemase Klebsiella pneumoniae with a Galleria mellonella model and a pilot study to translate to patient outcomes. BMC Infect. Dis. 2014, 14, 31. [CrossRef]

21. Malanovic, N.; Lohner, K. Antimicrobial peptides targeting Gram-positive bacteria. Pharmaceuticals (Basel) 2016, 9, 59. [CrossRef] [PubMed]

22. Lohner, K. New strategies for novel antibiotics: Peptides targeting bacterial cell membranes. Gen. Physiol. Biophys. 2009, 28, 105-116. [CrossRef] [PubMed]

23. Loh, J.M.; Adenwalla, N.; Wiles, S.; Proft, T. Galleria mellonella larvae as an infection model for group A streptococcus. Virulence 2013, 4, 419-428. [CrossRef] [PubMed]

24. Carmona-Ribeiro, A.M.; de Melo Carrasco, L.D. Cationic antimicrobial polymers and their assemblies. Int. J. Mol. Sci. 2013, 14, 9906-9946. [CrossRef] [PubMed]

25. Mahlapuu, M.; Håkansson, J.; Ringstad, L.; Björn, C. Antimicrobial peptides: An emerging category of therapeutic agents. Front Cell Infect. Microbiol. 2016, 6, 194. [CrossRef] [PubMed]

26. Kaplan, J.B.; Velliyagounder, K.; Ragunath, C.; Rohde, H.; Mack, D.; Knobloch, J.K.; Ramasubbu, N. Genes involved in the synthesis and degradation of matrix polysaccharide in Actinobacillus actinomycetemcomitans and Actinobacillus pleuropneumoniae biofilms. J. Bacteriol. 2004, 186, 8213-8220. [CrossRef]

27. Matsuzaki, K. Why and how are peptide-lipid interactions utilized for self-defense? Magainins and tachyplesins as archetypes. Biochimica Biophysica Acta (BBA) Biomembranes 1999, 1462, 1-10. [CrossRef]

28. Kaplan, C.W.; Sim, J.H.; Shah, K.R.; Kolesnikova-Kaplan, A.; Shi, W.; Eckert, R. Selective membrane disruption: Mode of action of C16G2, a specifically targeted antimicrobial peptide. Antimicrob. Agents Chemother. 2011, 55, 3446-3452. [CrossRef]

29. Wimley, W.C. Describing the mechanism of antimicrobial peptide action with the interfacial activity model. ACS Chem. Biol. 2010, 5, 905-917. [CrossRef]

30. World Health Organization. Global Priority List of Antibiotic-Resistant Bacteria to Guide Research, Discovery, and Development of New Antibiotics. 2017. Available online: https://www.who.int/medicines/publications/global-priority-list-antibioticresistant-bacteria/en (accessed on 6 January 2021).

31. Palermo, E.F.; Lienkamp, K.; Gillies, E.R.; Ragogna, P.J. Antibacterial activity of polymers: Discussions on the nature of amphiphilic balance. Angew. Chem. Int. Ed. 2019, 58, 3690-3693. [CrossRef]

32. Kenawy, E.-R.; Worley, S.D.; Broughton, R. The chemistry and applications of antimicrobial polymers: A state-of-the-art review. Biomacromolecules 2007, 8, 1359-1384. [CrossRef]

33. Wang, G.; Li, X.; Wang, Z. APD3: The antimicrobial peptide database as a tool for research and education. Nucleic Acids Res. 2016, 44, D1087-D1093. [CrossRef]

34. Kuroda, K.; Caputo, G.A.; DeGrado, W.F. The role of hydrophobicity in the antimicrobial and hemolytic activities of polymethacrylate derivatives. Chemistry 2009, 15, 1123-1133. [CrossRef]

35. Palermo, E.F.; Kuroda, K. Chemical structure of cationic groups in amphiphilic polymethacrylates modulates the antimicrobial and hemolytic activities. Biomacromolecules 2009, 10, 1416-1428. [CrossRef]

36. Palermo, E.F.; Lee, D.K.; Ramamoorthy, A.; Kuroda, K. Role of cationic group structure in membrane binding and disruption by amphiphilic copolymers. J. Phys. Chem. B 2011, 115, 366-375. [CrossRef]

37. Al-Badri, Z.M.; Som, A.; Lyon, S.; Nelson, C.F.; Nusslein, K.; Tew, G.N. Investigating the effect of increasing charge density on the hemolytic activity of synthetic antimicrobial polymers. Biomacromolecules 2008, 9, 2805-2810. [CrossRef]

38. Lin, J.; Qiu, S.; Lewis, K.; Klibanov, A.M. Bactericidal properties of flat surfaces and nanoparticles derivatized with alkylated polyethylenimines. Biotechnol. Prog. 2002, 18, 1082-1086. [CrossRef]

39. Curtis, K.A.; Miller, D.; Millard, P.; Basu, S.; Horkay, F.; Chandran, P.L. Unusual salt and pH induced changes in polyethylenimine solutions. PLOS ONE 2016, 11, e0158147. [CrossRef]

40. Liu, L.; Xu, K.; Wang, H.; Tan, P.K.; Fan, W.; Venkatraman, S.S.; Li, L.; Yang, Y.Y. Self-assembled cationic peptide nanoparticles as an efficient antimicrobial agent. Nat. Nanotechnol. 2009, 4, 457-463. [CrossRef]

41. Yasir, M.; Dutta, D.; Willcox, M.D.P. Comparative mode of action of the antimicrobial peptide melimine and its derivative Mel4 against Pseudomonas aeruginosa. Sci. Rep. 2019, 9, 7063. [CrossRef]

42. Kwon, J.Y.; Kim, M.K.; Mereuta, L.; Seo, C.H.; Luchian, T.; Park, Y. Mechanism of action of antimicrobial peptide P5 truncations against Pseudomonas aeruginosa and Staphylococcus aureus. AMB Express. 2019, 9, 122. [CrossRef]

43. Bengoechea, J.A.; Sa Pessoa, J. Klebsiella pneumoniae infection biology: Living to counteract host defences. FEMS Microbiol. Rev. 2019, 43, 123-144. [CrossRef]

44. Fleeman, R.M.; Macias, L.A.; Brodbelt, J.S.; Davies, B.W. Defining principles that influence antimicrobial peptide activity against capsulated Klebsiella pneumoniae. Proc. Natl. Acad. Sci. USA 2020, 117, 27620. [CrossRef] 
45. Liu, X.; Tang, M.; Zhang, T.; Hu, Y.; Zhang, S.; Kong, L.; Xue, Y. Determination of a threshold dose to reduce or eliminate CdTe-induced toxicity in L929 cells by controlling the exposure dose. PLoS ONE 2013, 8, e59359. [CrossRef]

46. Venkatesh, M.; Barathi, V.A.; Goh, E.T.L.; Anggara, R.; Fazil, M.; Ng, A.J.Y.; Harini, S.; Aung, T.T.; Fox, S.J.; Liu, S.; et al. Antimicrobial activity and cell selectivity of synthetic and biosynthetic cationic polymers. Antimicrob. Agents Chemother. 2017, 61. [CrossRef]

47. Aguiar-Ricardo, A.; Bonifácio, V.D.B.; Casimiro, T.; Correia, V.G. Supercritical carbon dioxide design strategies: From drug carriers to soft killers. Philos. Trans. A Math Phys. Eng. Sci. 2015, 373. [CrossRef]

48. Burckhardt, I.; Zimmermann, S. Susceptibility testing of bacteria using Maldi-Tof Mass Spectrometry. Front. Microbiol. 2018, 9 , 1744. [CrossRef]

49. Edwards-Jones, V.; Claydon, M.A.; Evason, D.J.; Walker, J.; Fox, A.J.; Gordon, D.B. Rapid discrimination between methicillinsensitive and methicillin-resistant Staphylococcus aureus by intact cell mass spectrometry. J. Med. Microbiol. 2000, 49, 295-300. [CrossRef]

50. Wiegand, I.; Hilpert, K.; Hancock, R.E. Agar and broth dilution methods to determine the minimal inhibitory concentration (MIC) of antimicrobial substances. Nat. Protoc. 2008, 3, 163-175. [CrossRef]

51. Pankey, G.A.; Sabath, L.D. Clinical relevance of bacteriostatic versus bactericidal mechanisms of action in the treatment of Gram-positive bacterial infections. Clin. Infect. Dis. 2004, 38, 864-870. [CrossRef]

52. García-Armesto, M.R.; Prieto, M.; García-López, M.L.; Otero, A.; Moreno, B. Modern microbiological methods for foods: Colony count and direct count methods. A review. Microbiologia 1993, 9, 1-13.

53. Mangoni, M.L.; Papo, N.; Barra, D.; Simmaco, M.; Bozzi, A.; Di Giulio, A.; Rinaldi, A.C. Effects of the antimicrobial peptide temporin L on cell morphology, membrane permeability and viability of Escherichia coli. Biochem. J. 2004, 380, 859-865. [CrossRef]

54. Xu, M.; McCanna, D.J.; Sivak, J.G. Use of the viability reagent PrestoBlue in comparison with alamarBlue and MTT to assess the viability of human corneal epithelial cells. J. Pharmacol. Toxicol. Methods 2015, 71, 1-7. [CrossRef]

55. Pinheiro, M.; Lúcio, M.; Lima, J.L.; Reis, S. Liposomes as drug delivery systems for the treatment of TB. Nanomedicine (Lond.) 2011, 6, 1413-1428. [CrossRef] 